\title{
Ocular blood flow values measured by laser speckle flowgraphy correlate with the postmenstrual age of normal neonates
}

\author{
Tadashi Matsumoto $^{1} \cdot$ Takashi Itokawa $^{1} \cdot$ Tomoaki Shiba $^{1}$ - Yuuji Katayama ${ }^{1} \cdot$ \\ Tetsushi Arimura ${ }^{1} \cdot$ Kotaro Hine $^{2}$ • Norio Mizukaki ${ }^{2} \cdot$ Hitoshi Yoda $^{2} \cdot$ Yuichi Hori $^{1}$
}

Received: 29 February 2016/Revised: 11 April 2016/Accepted: 18 April 2016/Published online: 27 April 2016

(C) The Author(s) 2016. This article is published with open access at Springerlink.com

\begin{abstract}
Purpose To evaluate the relationships between optic nerve head $(\mathrm{ONH})$ blood flow by laser speckle flowgraphy (LSFG), and postmenstrual age and body weight in normal neonates.

Methods During their normal sleep, we studied 24 infants (postmenstrual age, 248-295 days) whose ocular blood flow could be measured three consecutive times. While the subjects slept in the supine position, three mean blur rate (MBR) values of the ONH were obtained: the MBR-A (mean of all values), MBR-V (vessel mean) and MBR-T (tissue mean) in the ONH. With regard to eye diseases, no retinopathy of prematurity (ROP) was observed, and no severe systemic diseases requiring treatment were noted in the subjects. Pearson's correlation coefficients were used to determine the relationship between the MBR-A, $-\mathrm{V},-\mathrm{T}$ and postmenstrual age (days) and body weight $(\mathrm{g})$.

Results Postmenstrual age was significantly correlated with MBR-A $(r=0.64, p=0.0007)$, MBR-V $(r=0.62, p=0.0012)$ and MBR-T $(r=0.62, p=0.0012)$. However, the body weight was not correlated with the MBR (MBR-A: $r=0.37, p=0.07$, MBR-V: $r=0.31, p=0.14$, MBR-T: $r=0.38, p=0.06$ ). Conclusions Our results clarified that the values of ocular blood flow measured by LSFG correlate with the postmenstrual age of normal neonates.
\end{abstract}

Tadashi Matsumoto

matsu-t@med.toho-u.ac.jp

1 Department of Ophthalmology, School of Medicine, Toho University, 6-11-1 Omori-Nishi, Ota-ku, Tokyo 143-8541, Japan

2 Department of Neonatology, School of Medicine, Toho University, Tokyo, Japan
Keywords Laser speckle flowgraphy · Optic nerve head . Ocular blood flow · Normal neonate $\cdot$ Postmenstrual age . LSFG-baby

\section{Introduction}

Laser speckle flowgraphy (LSFG) can be used to noninvasively measure ocular blood flow [1-4]. LSFG is based on the changes in the speckle pattern of laser light reflected from the eye [5], and since LSFG is dependent on the movement of erythrocytes in the retina and choroid [6], it can measure the relative velocity of the erythrocytes' mean blur rate (MBR). LSFG is used for research on various diseases in adult patients, such as glaucoma [7, 8], retinal vein occlusion [9] and diabetic retinopathy [10]. It is also used in research related to aging and retinal blood flow changes [11-13]. Ocular diseases in neonates such as the retinopathy of prematurity (ROP) changes in retinal circulation occur with dilation and tortuosity of retinal blood vessels [14].

Until now, studies of the neonatal ophthalmic artery (OA) and central retinal artery (CRA) have been made using Color Doppler imaging (CDI) [15-20]. However, there has been no study taking direct measurements of the intraocular blood flow, and the relation between ROP and the ocular blood flow is still unknown. We have nevertheless speculated that the relationships between the ocular blood flow and neonatal ocular diseases could be clarified by researching retinal hemodynamics in neonates. We therefore measured the ocular blood flow in neonates using a version of LSFG modified for use in neonate patients (i.e., the LSFG-baby system), and we observed that the reproducibility of the results obtained with LSFG-baby is good [21].

The details of neonatal ocular blood flow changes are not known, but it is known that (1) the amount of general 
circulation in neonates is related to the neonatal body weight (g), and (2) the cerebral blood volume (CBV) is related to the neonate's postmenstrual age (days) [22]. For this reason, we also considered the possibility that the neonatal ocular blood flow is correlated with changes in body weight and postmenstrual age, and we conducted the present study to test this possibility.

We measured the ocular circulation of the optic nerve head $(\mathrm{ONH})$ in normal neonates using LSFG-baby, and we assessed the relationships between these blood flow values and the body weight and postmenstrual age. Our findings thus define the standard values of LSFG and indices in the early neonatal period, and our results provide the first step in ocular circulation studies of ROP.

\section{Subjects and methods}

\section{Subjects}

The subjects for our investigation were non-incubator neonates who were not on respirators. For each subject, an ophthalmologist had requested an examination due to suspicion of ROP or other diseases between March 2015 and December 2015 at the Toho University Omori Medical Center. We studied 24 neonates ( 24 eyes) during sleep in whom the circulation could be measured three times consecutively at the initial test (11 males, 13 females; postmenstrual age: 248-295 days, i.e., 34-42 weeks). With regard to eye diseases, no ROP was identified, and no severe systemic diseases requiring treatment were noted in the subjects.

This study was conducted in accord with the principles laid out in the Declaration of Helsinki, and the data analysis was approved by the ethical review committee of Toho University (nos. \#26-96, \#27-19).

\section{LSFG measurement}

Measurements were obtained using the 'LSFG-baby' system [21], which is a version of the commercially available LSFGNAVI system (Softcare, Fukuoka, Japan) modified in such a manner that the testing can be performed with the neonate in the supine position (Fig. 1). The testing followed previously reported methods [21]. Briefly, pupillary dilation was achieved with $2.625 \%$ phenylephrine hydrochloride, $0.125 \%$ tropicamide and $0.25 \%$ cyclopentolate, after which the $\mathrm{ONH}$ was imaged for $3 \mathrm{sec}$. Testing was performed while the infant was sleeping to measure his or her circulation during rest. Without the use of a lid speculum, the tester gently held the infant's eyelid open with a finger during the examination. All measurements were performed by the same tester. The testing was concluded within $10 \mathrm{~min}$, and measured only the left eye. After testing, the images were confirmed, and results

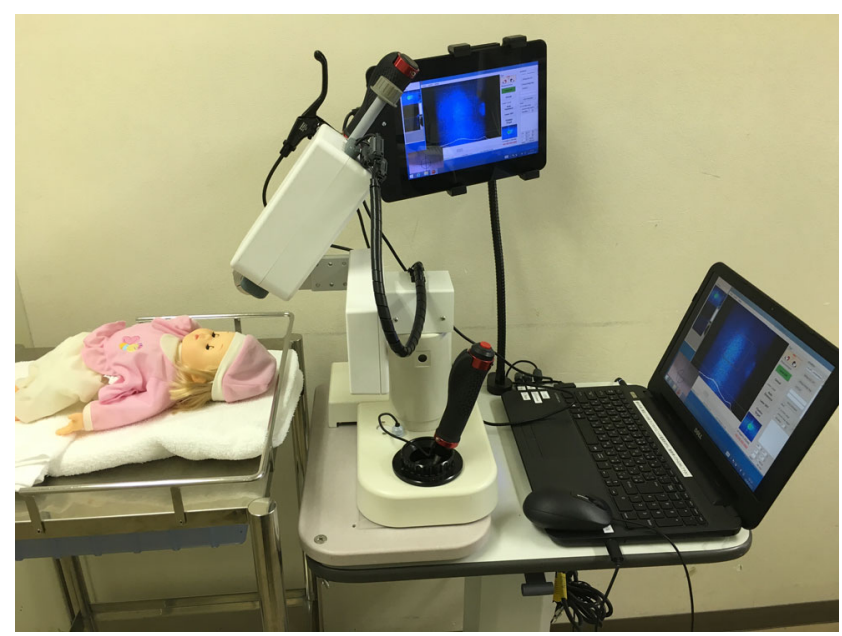

Fig. 1 The LSFG-baby system. Examination of a neonate in the supine position. The system's camera is set on a tilting stage with two axes ( $\varphi$ and $\theta$ ) and $x-y$ stages to adjust the field of view

that were significantly out of focus, or for which only two heart beats or less were measured, were excluded.

\section{Measurements of systemic parameters and other ocular parameters}

The following parameters were measured: systolic blood pressure (SBP, mmHg), diastolic blood pressure (DBP, $\mathrm{mmHg}$ ), pulse pressure $(\mathrm{mmHg})$, heart rate (beats per min, bpm), and intraocular pressure (IOP, $\mathrm{mmHg}$ ) measured by a Tono-Pen Avia (RE Medical, Osaka, Japan). We calculated the mean arterial blood pressure (MABP, $\mathrm{mmHg}$ ) and ocular perfusion pressure (OPP, $\mathrm{mmHg}$ ) with the following formulas. The MABP was determined by the formula: $\mathrm{DBP}+(\mathrm{SBP}$ $-\mathrm{DBP}) / 3)$. The OPP was defined as: (2/3MABP)-IOP. All parameters were evaluated after the LSFG measurements were obtained.

\section{Analysis of the MBR values of the ONH}

We analyzed the MBR by setting a rubber band on the $\mathrm{ONH}$ [23] (Fig. 2). The three MBR parameters of MBR-A (the mean of all values), MBR-V (the vessel mean) and MBR-T (the tissue mean) were analyzed by the LSFG Analyzer software (Softcare). Each result was a mean of three measurements. For the evaluation of the reproducibility of the measurement method, the analysis was performed using the MBR of the $\mathrm{ONH}$ with which the coefficient of variation (CV) and intraclass correlation coefficient (ICC) were measured three consecutive times.

\section{Statistical analysis}

Data are presented as the mean \pm standard deviation (SD) for the continuous variables. Pearson's correlation coefficients 


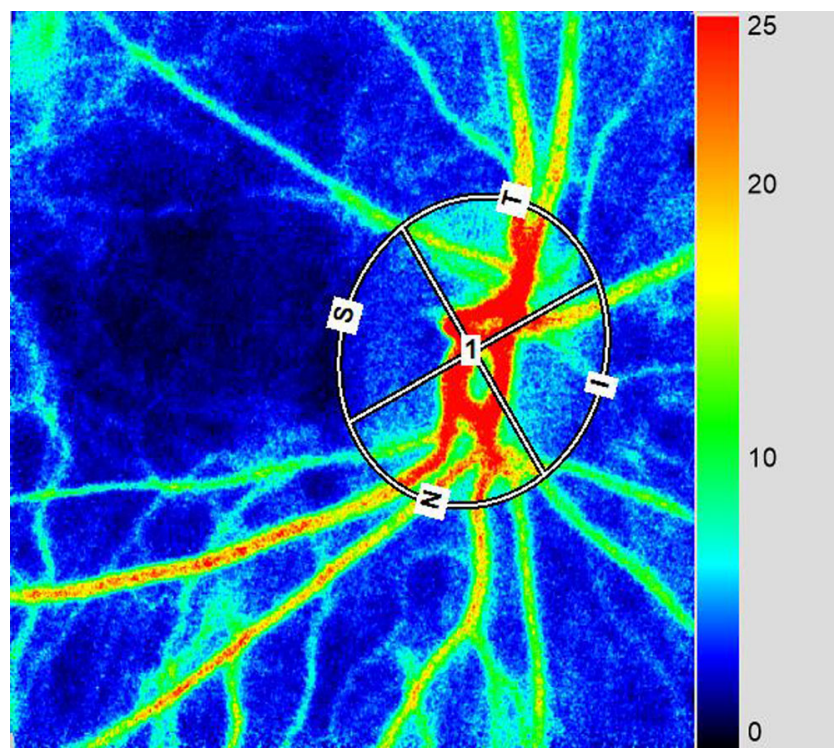

Fig. 2 In the color-coded maps, red indicates high blood flow and blue indicates low blood flow. The image is observed indirectly

were used to determine the relationship between the MBR-A, MBR-V, MBR-T and postmenstrual age, body weight, and other systemic and ocular parameters. $P$ values $<0.05$ were considered significant. The data were analyzed using the 12.1.0 version of the statistical software JMP (SAS, Cary, $\mathrm{NC})$.

\section{Results}

Table 1 shows the characteristics of the 24 subjects. Table 2 shows the CVs and ICCs for the MBR of the ONH values of the 24 subjects. All CVs from these patients were $<10 \%$, and all ICC results were $>0.8$. The MBR-A was $11.2 \pm 3.3$ (range 5.7-17.7), the MBR-V was $21.5 \pm 5.9$ (range 11.7-30.5) and the MBR-T was $7.9 \pm 2.5$ (range 3.5-14.2).

The Pearson's correlation coefficients between the MBR of the $\mathrm{ONH}$ and postmenstrual age and body weight are given in Table 3. Postmenstrual age was significantly correlated with the MBR-A $(r=0.64, p=0.0007)$, MBR-V $(r=0.62$, $p=0.0012)$ and MBR-T $(r=0.62, p=0.0012)$ (Fig. 3a). However, the body weight was not correlated with the MBR (MBR-A: $r=0.37, p=0.07$; MBR-V: $r=0.31, p=0.14$; MBRT: $r=0.38, p=0.06$; Fig. $3 b$ ).

In addition, the following parameters were not correlated with any aspect of the MBR: gestational age, chronologic age, birth weight, heart rate and OPP.

\section{Discussion}

This study is the first to examine the blood flow in normal neonates using LSFG. We measured the ocular blood flow in
Table 1 Characteristics of the 24 neonates

\begin{tabular}{lc}
\hline & Mean \pm SD \\
\hline Postmenstrual age (days) & $267.5 \pm 13.9$ \\
Gestational age (days) & $237.7 \pm 21.2$ \\
Chronologic age (days) & $29.8 \pm 17.2$ \\
Body weight (g) & $2245.3 \pm 387.3$ \\
Birth weight (g) & $1867.1 \pm 632.3$ \\
Heart rate (bpm) & $140.0 \pm 14.1$ \\
MABP (mmHg) & $47.8 \pm 5.3$ \\
IOP (mmHg) & $15.4 \pm 2.4$ \\
OPP (mmHg) & $16.5 \pm 4.0$ \\
Gender (m:f) & $11: 13$ \\
\hline
\end{tabular}

$M A B P$ mean arterial blood pressure, IOP intraocular pressure, $O P P$ ocular perfusion pressure

24 neonates, and our findings revealed a correlation between the MBR of the ONH and postmenstrual age. No correlation was observed between the MBR and body weight, gestational age, chronological age, birth weight or OPP. The reproducibility of the results was excellent, with a $\mathrm{CV}$ of not more than $10 \%$ and an ICC of not less than 0.8 , as reported earlier [21].

The correlation between the volume of the general circulation of neonates and their body weight is generally understood, and the body weight is used for calculating the general circulation volume. However, it has been reported that the cerebral circulation volume expressed as CBV correlates more strongly with corrected age in days than with body weight [22]. The growth of the head, including the eyeballs, is generally completed sooner than the development of the whole body, which may be related to the results of this study. In other words, it is likely that the mean blur rate of the $\mathrm{ONH}$ is involved in eye development in terms of function due to changes in the age in days rather than changes in whole-body growth and circulation.

Earlier studies of ocular blood flow in neonates by CDI showed an increase in the blood flow velocity in the OA $[15,17]$ and CRA $[17,20]$ along with age in days. However, to our knowledge, there is no study on the correlation of these blood flow velocities with postmenstrual age or body weight. Further, CDI determines the absolute value of blood flow

Table 2 Coefficients of variation $(\mathrm{CV})$ and intraclass correlation coefficient (ICC) for ONH blood flow values $(n=24)$

\begin{tabular}{lllr}
\hline & CV $(\%)$ & ICC & Mean \pm SD \\
\hline MBR-A & $8.3 \pm 3.1$ & 0.87 & $11.2 \pm 3.3$ \\
MBR-V & $9.8 \pm 4.6$ & 0.80 & $21.5 \pm 5.9$ \\
MBR-T & $9.2 \pm 4.1$ & 0.87 & $7.9 \pm 2.5$ \\
\hline
\end{tabular}

$M B R$ mean blur rate, $M B R-A$ mean of all values, $M B R-V$ vessel mean, $M B R-T$ tissue mean 
Table 3 Pearson's correlation coefficients between MBR (-A, $-\mathrm{V}$, and $-\mathrm{T})$ and all parameters $(n=24)$

\begin{tabular}{|c|c|c|c|c|c|c|}
\hline \multirow[t]{2}{*}{ Explanatory variable } & \multicolumn{2}{|c|}{ MBR-A } & \multicolumn{2}{|c|}{ MBR-V } & \multicolumn{2}{|c|}{ MBR-T } \\
\hline & $r$ & $p$ & $r$ & $p$ & $r$ & $p$ \\
\hline Postmenstrual age (days) & 0.64 & 0.0007 & 0.62 & 0.0012 & 0.62 & 0.0012 \\
\hline Gestational age (days) & 0.23 & 0.29 & 0.22 & 0.31 & 0.21 & 0.32 \\
\hline Chronologic age (day) & 0.24 & 0.25 & 0.24 & 0.26 & 0.24 & 0.26 \\
\hline Body weight (g) & 0.37 & 0.07 & 0.31 & 0.14 & 0.38 & 0.06 \\
\hline Birth weight (g) & 0.08 & 0.72 & 0.05 & 0.83 & 0.07 & 0.74 \\
\hline Heart rate (bpm) & 0.02 & 0.94 & -0.08 & 0.70 & 0.15 & 0.49 \\
\hline OPP (mmHg) & -0.13 & 0.55 & -0.09 & 0.69 & -0.15 & 0.47 \\
\hline
\end{tabular}

$M B R$ mean blur rate, $M B R-A$ mean of all values, $M B R-V$ vessel mean, $M B R-T$ tissue mean, $O P P$ ocular perfusion pressure

velocity, but this may not reflect the changes in the blood flow volume if the blood vessel diameter has changed.

On the other hand, in studies that used LSFG, since the blood flow value is determined by assigning a rubber band directly to the ONH [21], LSFG is thought to be advantageous for neonatal blood flow measurement. In addition, unlike CDI, LSFG does not provide the absolute value of the blood flow volume, and making comparisons between individuals
Fig. 3 Correlations between MBR and postmenstrual age (days) or body weight (g). a Relationships between MBR ( $-\mathrm{A}$ [all values' mean], $-\mathrm{V}$ [vessel mean], $-\mathrm{T}$ [tissue mean]) and postmenstrual age. b Relationships between $\operatorname{MBR}(-\mathrm{A},-\mathrm{V},-\mathrm{T})$ and body weight a

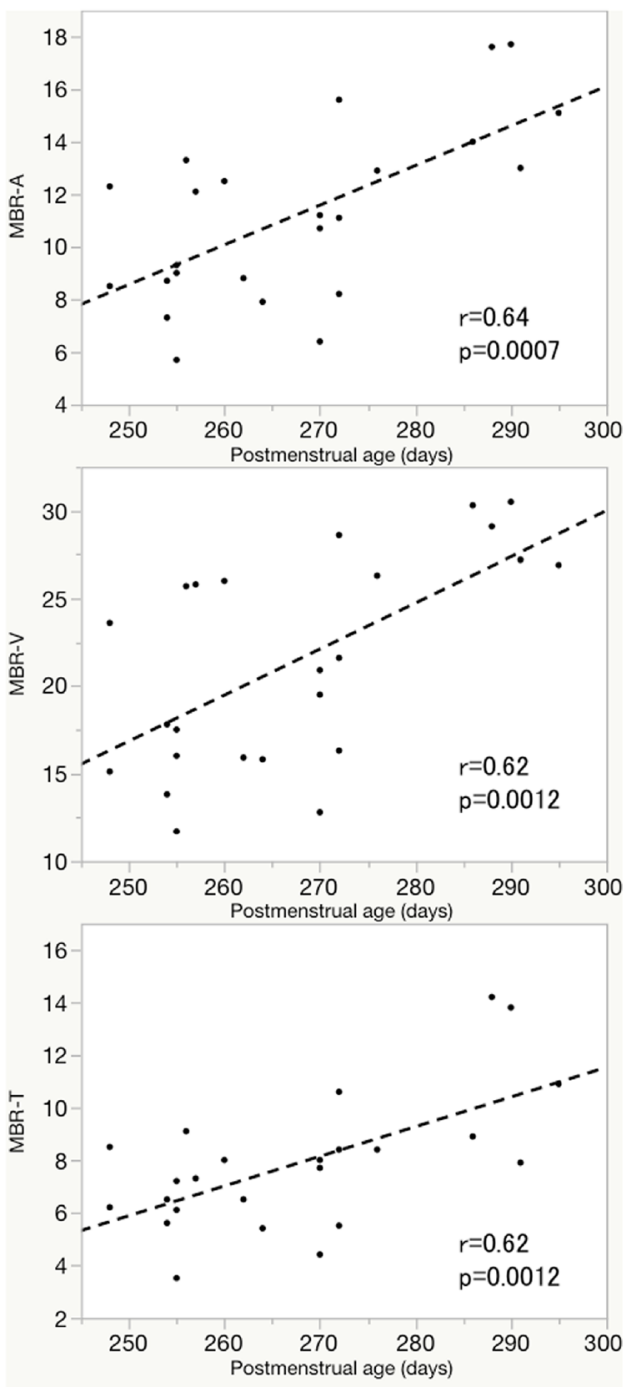

b

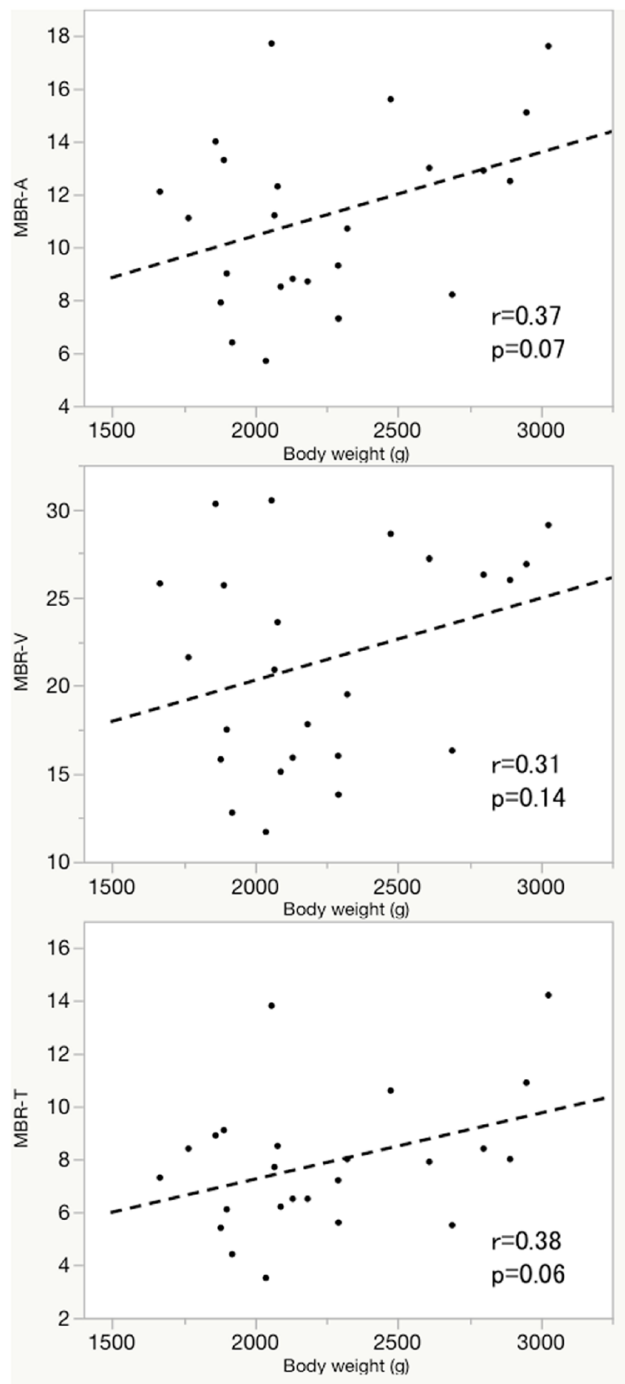


with LSFG is said to be difficult [23]. However, Aizawa et al. [24] are of the opinion that the MBR of the ONH determined by LSFG and the values measured using the hydrogen clearance method correlate well under almost identical conditions, and therefore can be used for comparing the MBR of the $\mathrm{ONH}$ between individuals.

The MBR for the normal neonates' ONHs in this study was lower compared to that for adults (age $60.4 \pm 12.5$ years, $n=93$ ) [25] who we examined: MBR-A, 21.9 \pm 3.8 ; MBR-V, 42.1 \pm 4.8 ; MBR-T, $11.9 \pm 1.9$. However, due to major differences between adults and neonates with regard to heart rate, blood pressure, OPP, and eye structure, a simple comparison is difficult.

This study has some limitations. First, examinations of the neonates, unlike adults, are difficult. The reproducibility of the examinations is also slightly inferior compared to the results of adults [7]. Second, the pupil diameter of a sleeping neonate is small, and therefore the examination is impossible without mydriasis. Third, due to the noninvasive nature of this study with natural sleeping neonates, observations of neonates who are under artificial ventilation in an incubator or infants who grew and were discharged from the hospital would be difficult with the LSFG-baby system. However, with further improvements to LSFG measurement devices, the number of test subjects will increase along with the accumulation of data, and studies in such subjects could become possible.

Here, we measured the mean blur rate and the neonatal ocular circulation. The results of our study suggest that neonatal ocular circulation measurements could become an indicator of eyeball function and retinal circulation growth, and our findings a first step in the study of the correlation of eye diseases and systemic diseases with ocular blood flow in neonates.

Acknowledgments This work was supported by Toho University project grants (\#26-34), a Toho University Nukada scholarship and a Toho University Shibata scholarship.

\section{Compliance with ethical standards}

Funding No funding was received for this research.

Conflicts of Interest The authors have no conflicts of interest to declare.

Ethical approval This study was conducted in accord with the principles laid out in the Declaration of Helsinki, and the ethical review committee of Toho University (\#26-96, \#27-19) approved the study.

Informed consent We began research after a parent of each subject provided informed consent.

Open Access This article is distributed under the terms of the Creative Commons Attribution 4.0 International License (http:// creativecommons.org/licenses/by/4.0/), which permits unrestricted use, distribution, and reproduction in any medium, provided you give appropriate credit to the original author(s) and the source, provide a link to the Creative Commons license, and indicate if changes were made.

\section{References}

1. Tamaki Y, Araie M, Kawamoto E, Eguchi S, Fujii H (1994) Noncontact, two-dimensional measurement of retinal microcirculation using laser speckle phenomenon. Invest Ophthalmol Vis Sci 35:3825-3834

2. Tamaki Y, Araie M, Kawamoto E, Eguchi S, Fujii H (1995) Noncontact, two-dimensional measurement of tissue circulation in choroid and optic nerve head using laser speckle phenomenon. Exp Eye Res 60:373-383

3. Tamaki Y, Araie M, Tomita K, Nagahara M, Tomidokoro A, Fujii H (1997) Real-time measurement of human optic nerve head and choroid circulation, using the laser speckle phenomenon. Jpn J Ophthalmol 41:49-54

4. Sugiyama T, Araie M, Riva CE, Schmetterer L, Orgul S (2010) Use of laser speckle flowgraphy in ocular blood flow research. Acta Ophthalmol 88:723-729. doi:10.1111/j.1755-3768.2009.01586.x

5. Isono H, Kishi S, Kimura Y, Hagiwara N, Konishi N, Fujii H (2003) Observation of choroidal circulation using index of erythrocytic velocity. Arch Ophthalmol 121:225-231

6. Fujii H (1994) Visualisation of retinal blood flow by laser speckle flowgraphy. Med Biol Eng Comput 32:302-304

7. Aizawa N, Yokoyama Y, Chiba N, Omodaka K, Yasuda M, Otomo T, Nakamura M, Fuse N, Nakazawa T (2011) Reproducibility of retinal circulation measurements obtained using laser speckle flowgraphy-NAVI in patients with glaucoma. Clin Ophthalmol 5: 1171-1176. doi:10.2147/OPTH.S22093

8. Shiga Y, Omodaka K, Kunikata H, Ryu M, Yokoyama Y, Tsuda S, Asano T, Maekawa S, Maruyama K, Nakazawa T (2013) Waveform analysis of ocular blood flow and the early detection of normal tension glaucoma. Invest Ophthalmol Vis Sci 54:76997706. doi:10.1167/iovs.13-12930

9. Maeda K, Ishikawa F, Ohguro H (2009) Ocular blood flow levels and visual prognosis in a patient with nonischemic type central retinal vein occlusion. Clin Ophthalmol 3:489-491

10. Enaida H, Okamoto K, Fujii H, Ishibashi T (2010) LSFG findings of proliferative diabetic retinopathy after intravitreal injection of bevacizumab. Ophthalmic Surg Lasers Imaging 41(Online):e1-e3

11. Shiba T, Takahashi M, Hori Y, Maeno T (2012) Pulse-wave analysis of optic nerve head circulation is significantly correlated with brachial-ankle pulse-wave velocity, carotid intima-media thickness, and age. Graefes Arch Clin Exp Ophthalmol 250:1275-1281. doi: 10.1007/s00417-012-1952-5

12. Shiba T, Takahashi M, Hori Y, Maeno T, Shirai K (2012) Optic nerve head circulation determined by pulse wave analysis is significantly correlated with cardio ankle vascular index, left ventricular diastolic function, and age. J Atheroscler Thromb 19:999-1005

13. Tsuda S, Kunikata H, Shimura M, Aizawa N, Omodaka K, Shiga Y, Yasuda M, Yokoyama Y, Nakazawa T (2014) Pulse-waveform analysis of normal population using laser speckle flowgraphy. Curr Eye Res 39:1207-1215. doi:10.3109/02713683.2014.905608

14. Chen J, Smith LE (2007) Retinopathy of prematurity. Angiogenesis 10:133-140. doi:10.1007/s10456-007-9066-0

15. Baerts W, Wildervanck de Blecourt-Devilee M, Sauer PJ (1993) Ambient light, ophthalmic artery blood flow velocities and retinopathy of prematurity. Acta Paediatr 82:719-722

16. Holland DR, Saunders RA, Kagemann LE, Bluestein EC, Hutchinson AK, Corson DW, Harris A (1999) Color doppler imaging of the central retinal artery in premature infants undergoing examination for retinopathy of prematurity. J AAPOS 3:194-198

17. Romagnoli C, Papacci P, Zecca E, Giannantonio C, De Carolis MP, Tortorolo G (2001) Normal neonatal values of ophthalmic and central retinal artery blood flow velocities. J Pediatr Ophthalmol Strabismus 38:213-217 
18. Niwald A, Gralek M (2006) Evaluation of blood flow in the ophthalmic artery and central retinal artery in children with retinopathy of prematurity. Klin Ocz 108:32-35

19. Neely D, Harris A, Hynes E, McNulty L, McCranor L, Siesky B, Plager D, Sprunger D, Roberts G (2009) Longitudinal assessment of plus disease in retinopathy of prematurity using color Doppler imaging. J AAPOS 13:509-511. doi:10.1016/j.jaapos.2009.08.012

20. Hartenstein S, Muller B, Metze B, Czernik C, Buhrer C (2015) Blood flow assessed by color Doppler imaging in retinopathy of prematurity. J Perinatol 35:745-747. doi:10.1038/jp.2015.45

21. Matsumoto T, Itokawa T, Shiba T, Katayama Y, Arimura T, Mizukaki N, Yoda H, Hori Y (2015) Reproducibility of neonate ocular circulation measurements using laser speckle flowgraphy. Biomed Res Int 2015:693056. doi:10.1155/2015/693056

22. Ijichi S, Kusaka T, Isobe K, Okubo K, Kawada K, Namba M, Okada H, Nishida T, Imai T, Itoh S (2005) Developmental changes of optical properties in neonates determined by near-infrared time- resolved spectroscopy. Pediatr Res 58:568-573. doi:10.1203/01. PDR.0000175638.98041.0E

23. Sugiyama T (2014) Basic technology and clinical applications of the updated model of laser speckle flowgraphy to ocular diseases. Photonics 1:220-234

24. Aizawa N, Nitta F, Kunikata H, Sugiyama T, Ikeda T, Araie M, Nakazawa T (2014) Laser speckle and hydrogen gas clearance measurements of optic nerve circulation in albino and pigmented rabbits with or without optic disc atrophy. Invest Ophthalmol Vis Sci 55:7991-7996. doi:10.1167/iovs. 14-15373

25. Shiba T, Sugiyama T, Hori Y, Matsumoto T, Maeno T, Takahashi M (2015) Relationship between plasma levels of vasoactive mediators and optic nerve head circulation shown by laser speckle flowgraphy. Graefes Arch Clin Exp Ophthalmol. doi:10.1007/ s00417-015-3145-5 